\title{
Scorpion primer PCR analysis for genotyping of allele variants of thiopurine s-methyltransferase*3
}

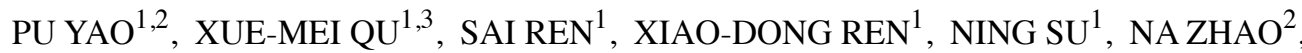 \\ LIU WANG $^{1}$, LIN CHENG $^{2}$, BANG-BI WENG ${ }^{2}$, FENG-JUN SUN ${ }^{2}$ and QING HUANG ${ }^{1}$ \\ ${ }^{1}$ Department of Laboratory Medicine, Daping Hospital, Third Military Medical University (Army Medical University), \\ Chongqing 400042; Departments of ${ }^{2}$ Pharmacy and ${ }^{3}$ Laboratory Medicine, Southwest Hospital, \\ Third Military Medical University (Army Medical University), Chongqing 400038, P.R. China
}

Received December 9, 2019; Accepted May 27, 2020

DOI: $10.3892 / \mathrm{mmr} .2020 .11283$

\begin{abstract}
Thiopurine S-methyltransferase (TPMT) plays an important role in the metabolism of thiopurines. Mutations in the TPMT gene can affect drug activity, which may have adverse effects in humans. Thus, genotyping can help elucidate genetic determinants of drug response to thiopurines and optimize the selection of drug therapies for individual patients, effectively avoiding palindromia during maintenance treatment caused by insufficient dosing and the serious side effects caused by excessive doses. The current available detection methods used for TPMT*3B and TPMT*3C are complex, costly and time-consuming. Therefore, innovative detection methods for TPMT genotyping are urgently required. The aim of the present study was to establish and optimize a simple, specific and timesaving TPMT genotyping method. Using the principles of Web-based Allele-Specific PCR and competitive real-time fluorescent allele-specific PCR (CRAS-PCR), two pairs of Scorpion primers were designed for the detection of TPMT*3B and $* 3 \mathrm{C}$, respectively, and a mutation in TPMT*3A
\end{abstract}

Correspondence to: Professor Qing Huang, Department of Laboratory Medicine, Daping Hospital, Third Military Medical University (Army Medical University), 10 Changjiang Branch Road, Chongqing 400042, P.R. China

E-mail: qinghunag@tmmu.edu.cn

Dr Feng-Jun Sun, Department of Pharmacy, Southwest Hospital, Third Military Medical University (Army Medical University), 30 Gaotanyan Street, Chongqing 400038, P.R. China

E-mail: fengj_sun@163.com

Abbreviations: TPMT, thiopurine S-methyltransferase; CRAS-PCR, competitive real-time fluorescent allele-specific PCR; 6-TG, 6-thioguanine; 6-MP, 6-thiopurine; AZA, azathioprine; 6-TGNs, 6-thioguanine nucleotides; SNPs, single nucleotide polymorphisms; WT, wild-type; MT, mutant; ASF, allele-specific forward; ASR, allele-specific reverse; COR, common reverse; COF, common forward; WASP, Web-based Allele-Specific PCR; RFU, relative fluorescence units

Key words: genotyping, TPMT*3A, TPMT*3B, TPMT*3C, Scorpion primer was inferred based on data from TPMT*3B and *3C. In total, 226 samples from volunteers living in Chongqing were used for CRAS-PCR to detect TPMT*3 mutations. Results showed that nine $(3.98 \%)$ were mutant $(\mathrm{MT})$ heterozygotes and none were MT homozygotes for TPMT*3C, and no TPMT*3A and TPMT*3B mutations were found. Three TPMT*3C MT heterozygotes were randomly selected for DNA sequencing, and CRAS-PCR results were consistent with the sequencing results. In conclusion, in order to improve simplicity, specificity and efficiency, the present study established and optimized CRAS-PCR assays for commonly found mutant alleles of TPMT*3A (G460A and A719G), TPMT*3B (G460A), and TPMT*3C (A719G).

\section{Introduction}

Thiopurines have been widely applied in clinical practice; among these, 6-thioguanine (6-TG), 6-thiopurine (6-MP) and its pro-drug azathioprine (AZA) are the most commonly used (1,2). 6-TG and 6-MP have been demonstrated to induce and maintain the stable stage of inflammatory bowel disease $(3,4)$ and are also the most widely used drugs in the treatment of children with acute lymphoblastic leukemia $(5,6)$. AZA plays an important role in the treatment of autoimmune diseases and acute rejection after organ transplantation (7). In vivo, thiopurines are catalyzed by metabolic enzymes into the active metabolite 6-TG nucleotides (6-TGNs), which participate in the synthesis of DNA and RNA molecules to exert cytotoxic and pharmacological effects by inhibiting DNA replication and RNA transcription as an antagonist of guanine nucleoside (8-10). In addition, thiopurines can also be metabolized by thiopurine S-methyltransferase (TPMT) into inactive 6-methyl-thioinosine monophosphate, which prevents the formation of 6-TGNs, thereby affecting the clinical efficacy and adverse drug reactions of thiopurines (8-10). Changes in TPMT activity are closely related to the concentration balance between 6-MP methyltransferase and 6-TGNs, and thus to the efficacy of thiopurine drugs $(2,11,12)$.

With research into the mechanism underlying the metabolic processing of thiopurine drugs, as well as the continuous disclosure of TPMT genetic polymorphisms, it has been revealed that TPMT genetic polymorphisms are single 
nucleotide polymorphisms (SNPs) that serve an important role in thiopurine therapies $(13,14)$. The pre-detection of TPMT genotypes should thus predict the toxicity of thiopurines and drug administration schedules can be adjusted accordingly. This serves to achieve individualized medicine and avoids palindromia during maintenance treatment caused by insufficient dosing and avoids the serious side effects caused by excessive doses. For patients lacking TPMT enzyme activity, other treatment options can also be considered. Numerous institutions and organizations $(15,16)$ have suggested that for patients who possess a heterozygous mutant (MT) TPMT genotype (i.e. intermediate activity), the initial dose of AZA or 6-MP should be reduced by $30-70 \%$. However, for patients who possess a homozygous MT TPMT genotype (i.e. MT variants with low or deficient activity), the initial dose of AZA, 6-MP or 6-TG should be reduced by 10 -fold, and an extended dosing frequency or alternative drugs should be selected. Therefore, TPMT genotyping should be considered in the process of thiopurine therapy, in order to tailor individual treatments to avoid toxicity and adverse reactions $(4,17,18)$.

With the development of gene detection technologies, the technologies for detection of gene polymorphisms include first-generation sequencing [i.e. DNA sequencing and quantitative PCR (qPCR)] (14,19-21) and next-generation sequencing (NGS; i.e. gene microarray and Genome Sequencer FLX) (22-24). Although direct sequencing remains the gold standard for the detection of SNPs, it also requires contact with potentially toxic chemicals (i.e. SYBR Green, Gold View, Gel Red or Acrylamide) or UV light for agarose gel electrophoresis, meaning there is still a risk of mutagenesis for the technician performing the sequencing, and the procedure and interpretation of results are time-consuming and labor-intensive. Therefore, it is necessary to develop methods for rapid and simple determination of SNPs without gene sequencing. NGS is high-throughput and has high accuracy. Moreover, NGS can be fully automated (22-24), allowing hundreds of thousands to millions of DNA molecules to be analyzed at a time, but it is not necessary for clinical patients for which only one or two SNPs require detection, and its use increases the financial burden on clinical patients. qPCR is a conventional gene detection technique that is convenient and quick to operate (14). Unfortunately, due to the thermodynamic driving force of thermophilic DNA polymerases, non-specific amplification commonly occurs $(25,26)$. These methods are complex, costly and time-consuming. Therefore, innovative detection methods for TPMT genotyping avoiding these shortcomings are urgently needed.

Our group previously delineated TPMT*2 mutations through competitive real-time fluorescent allele-specific PCR (CRAS-PCR) (19); the single-tube detection of the TPMT*2 polymorphism only required $1.5 \mathrm{~h}$. The relative fluorescence intensity is related to the gene copy number, meaning that results can be directly judged by amplification curves. In general, the CRAS-PCR method is more specific and sensitive than qPCR, particularly for large quantities of samples. Therefore, this study further developed a CRAS-PCR system targeting TPMT*3, which has higher MT frequency than TPMT*2. In this paper, based on the Web-based Allele-Specific PCR (WASP) principle (25), Scorpion primers suitable for polymorphism detection of TPMT*3B and $* 3 \mathrm{C}$ were designed.
Wild-type (WT)-allele-specific forward (ASF) and MT-ASF Scorpion primers were used for TPMT*3B detection in a single reaction mixture, and WT-allele-specific reverse (ASR) and MT-ASR Scorpion primers targeting TPMT*3C were used in another single reaction mixture. Application of this analysis to 226 samples confirmed that CRAS-PCR was suitable for genotyping TPMT*3B and $* 3 \mathrm{C}$ variants.

\section{Materials and methods}

Instruments and reagents. The traditional end-point PCR instrument used for the construction of the quality control (QC) plasmids was the C1000 Touch Thermal cycler (Bio-Rad Laboratories, Inc.). qPCR data were collected and analyzed using a ViiA ${ }^{\mathrm{TM}} 7$ Real-Time PCR system with a 96-well block (Applied Biosystems; Thermo Fisher Scientific, Inc.). DNA was collected using a QIAamp DNA Blood Mini kit (Qiagen $\mathrm{GmbH}$ ) and analyzed using a NanoDrop ND-1000 Spectrophotometer (NanoDrop; Thermo Fisher Scientific, Inc.).

Ex Taq DNA Polymerase Hot-Start Version and MutanBEST kit (Takara Biotechnology Co., Ltd.) were used to construct WT-QC and MT-QC plasmids. qPCR was performed with 2X Premix Ex Taq ${ }^{\mathrm{TM}}$ (Probe qPCR) master mix (Takara Biotechnology Co., Ltd.). QC plasmids were sequenced using a Big Dye Terminator V3.1 Cycler Sequencing kit (Applied Biosystems; Thermo Fisher Scientific, Inc.) on an ABI Prism 3500 Genetic Analyzer (Applied Biosystems; Thermo Fisher Scientific, Inc.). Oligonucleotides were synthesized at General Biosystems (Anhui) Corporation, Ltd.

Samples and genomic DNA extraction. The present study was carried out in accordance with the Declaration of Helsinki and was approved by the ethics committee of Southwest Hospital (Chongqing, China). In total, 226 samples (53.6\% male; age, $32 \pm 11$ years; all Han Chinese) were collected from healthy volunteers living in Chongqing. Before peripheral blood was collected, the volunteers were informed of the possible risks (i.e., pain, bleeding, redness and swelling) and signed informed consent documents. A peripheral blood sample was taken from each individual and added to a tube with EDTA before being identified with a unique code. Genomic DNA was extracted using the QIAamp DNA Blood Mini kit, according to the manufacturer's protocol. The genomic DNA concentration of each sample was quantified using a NanoDrop ND-1000 Spectrophotometer, following the manufacturer's instructions.

Construction of $Q C$ plasmids. The PCR reaction mixture (20 $\mu \mathrm{l})$ used to amplify whole exon 7 fragments containing TPMT*3B or exon 10 fragments containing TPMT*3C included 1X ExTaq Buffer $\left(\mathrm{Mg}^{2+}\right.$ plus), $250 \mu \mathrm{M}$ each dNTP, 0.5 units Ex Taq HS, $200 \mathrm{nM}$ each forward and reverse primer (Table I) and $10 \mathrm{ng}$ human genomic DNA. Reactions were performed under the following conditions: An initial denaturation at $95^{\circ} \mathrm{C}$ for $3 \mathrm{~min} ; 39$ cycles at $95^{\circ} \mathrm{C}$ for $30 \mathrm{sec}, 55^{\circ} \mathrm{C}$ for $30 \mathrm{sec}$ and $72^{\circ} \mathrm{C}$ for $30 \mathrm{sec}$; and final extension at $72^{\circ} \mathrm{C}$ for $5 \mathrm{~min}$. Sanger sequencing of the PCR products was performed using the BigDye Terminator V3.1 Cycler Sequencing kit. After the WT alleles of TPMT*3B and TPMT*3C were confirmed, the $\mathrm{PCR}$ products were cloned to construct the WT-QC plasmids. 
Table I. Oligonucleotides used in the present study.

\begin{tabular}{|c|c|c|}
\hline ID & Name & Sequence $\left(5^{\prime}-3^{\prime}\right)$ \\
\hline HQ-593 & Exon-7.F & CCTAATACCTTGACGATTGTTG \\
\hline HQ-594 & Exon-7.R & TAGAAGTCTAAGCTGATTTTCT \\
\hline HQ-611 & Exon-10.F & CCCTGATGTCATTCTTCATAG \\
\hline HQ-612 & Exon-10.R & GGCTTTAGCCATAATTTTCAAT \\
\hline HQ-1742 & TPMT*3B.WT-ASF & $\begin{array}{l}\text { (VIC) } \text { ACCGCGCGCGATCACCTGGATTGATGGCAACTAA } \\
\text { GCGCGGT(BHQ1)(HEG)ACATGATTTGGGATAGAGGTG }\end{array}$ \\
\hline HQ-1743 & TPMT*3B.MT-ASF & $\begin{array}{l}\text { (FAM) } \text { ACCGCGCGCGATCACCTGGATTGATGGCAACTA } \\
\text { AGCG CGGT(BHQ1)(HEG)GACATGATTTGGGATAGAG } \\
\text { GTA }\end{array}$ \\
\hline HQ-1744 & TPMT*3C.WT-ASR & $\begin{array}{l}\text { (VIC)ACCGCGCGAACGACATAAAAGTTGGGGAATTGA } \\
\text { CTGTCTTTTGCGCGGT(BHQ1)(HEG)CTCATTTACTTTT } \\
\text { CTGTAAGTAGCT }\end{array}$ \\
\hline HQ-1745 & TPMT*3C.MT-ASR & 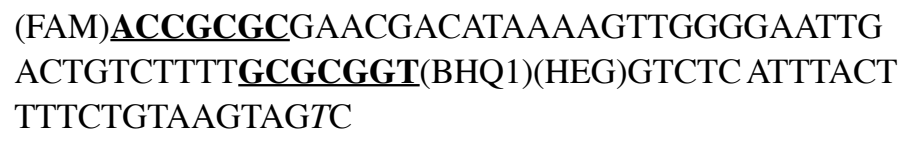 \\
\hline HQ-1608 & TPMT*3B.COR & CAAACTCATAGAAGTCTAAGCTGATTTTCT \\
\hline HQ-1609 & TPMT*3C.COF & CAATATACGTTGTCTTGAGAAGGTTGA \\
\hline
\end{tabular}

The positions of TPMT*3B and TPMT*3C in genomic DNA refer to the primer regions located in the TPMT reference genomic DNA(GenBank ID: NG_012137.2). HQ-1742 and HQ-1743 are Scorpion primers specifically targeting the WT and MT TPMT*3B alleles, whereas HQ-1744 and HQ-1745 are Scorpion primers for WT and MT TPMT*3C alleles. The stem-loop structure is marked with underlined bold letters. The position of the labeled fluorescent reporter (VIC or FAM), the corresponding quencher (BHQ1) and amplification blocker (HEG) are indicated. The italicized letters in the penultimate position indicate the mismatch introduced to increase AS primer specificity according to the WASP principle. The 3'-end terminal underlined letters indicate allele-distinguishable variants. AS, allele-specific; HEG, hexa-ethylene glycol; MT, mutant; WT, wild type; TPMT, TPMT, thiopurine S-methyltransferase; WASP, Web-based Allele-Specific PCR; F, forward; R, reverse.

The WT-QC plasmids of TPMT*3B and TPMT*3C were subsequently used to prepare the MT-QC plasmids using the MutanBEST kit, according to the manufacturer's instructions.

$q P C R$. Three types of primers were designed according to WASP principles (25) for TPMT*3B: WT-ASF and MT-ASF Scorpion primers, and a common reverse primer (Table I). Another three types of primers were designed for TPMT*3C: WT-ASR and MT-ASR Scorpion primers, and a common forward primer (Table I). The qPCR mixture $(20 \mu 1)$ for TPMT $* 3$ B or TPMT $* 3$ C contained 1 XPremix Ex Taq ${ }^{\mathrm{TM}}$, a fixed concentration of primers and QC plasmid (Tables SI and SII). Reactions were performed under the following cycling conditions: An initial denaturation at $95^{\circ} \mathrm{C}$ for $2 \mathrm{~min}$, followed by 50 cycles at $95^{\circ} \mathrm{C}$ for $15 \mathrm{sec}$ and at $59^{\circ} \mathrm{C}$ for $1 \mathrm{~min}$ (with double fluorescence acquisition). The quantification cycle $(\mathrm{Cq})$ values were automatically determined using the ViiA ${ }^{\mathrm{TM}} 7$ Real-Time PCR system with a 96-well block. All reactions were performed in duplicate with no-template controls to monitor for contamination and non-specific products. A 1:1 mixture of WT-QC and MT-QC plasmids was used as MIX-QC plasmids for TPMT*3B and TPMT*3C, respectively.

Principles of CRAS-PCR. A schematic diagram illustrating the protocol for CRAS-PCR is presented in our previous study (19). Following the WASP principles, two pairs of forward (or reverse) primers and a common reverse (or forward) primer were designed for TPMT*3B and TPMT*3C genotyping. The primers in the CRAS-PCR system are constructed in the shape of a scorpion structure, in which a unique fluorescent reporter (VIC or FAM) and a corresponding quencher (BH1) are labeled at the start and end of a stem-loop structure of the Scorpion primers separately (Table I). In the process of PCR extension, based on fluorescence resonance energy transfer (27), the DNA polymerase could not extend to the probe attached to the primer due to the steric resistance effect of 18 carbon atoms provided by hexa-ethylene glycol (28); thus, the integrity of the copied target DNA chain is ensured (Figs. 1 and 2).

Statistical analysis. qPCR data were analyzed by ABI 7500 v2.0 (Applied Biosystems; Thermo Fisher Scientific Inc.); the statistical threshold was automatically set by $\mathrm{ViiA}^{\mathrm{TM}} 7$ Real-Time PCR system, the difference in the Cq value between FAM and VIC was denoted $\Delta \mathrm{Cq}$ (29). Distribution frequencies of alleles and genotypes were analyzed by using a XY model of GraphPad Prism 5.01 software (GraphPad Software, Inc.), in which the fluorescence units of VIC and FAM of each reaction were inputted to automatically analyze and count the number of genotypes.

\section{Results}

Construction of TPMT*3B and $* 3 C$ QC-plasmids. The PCR products of the TPMT exon 7 and exon 10 were cloned to 


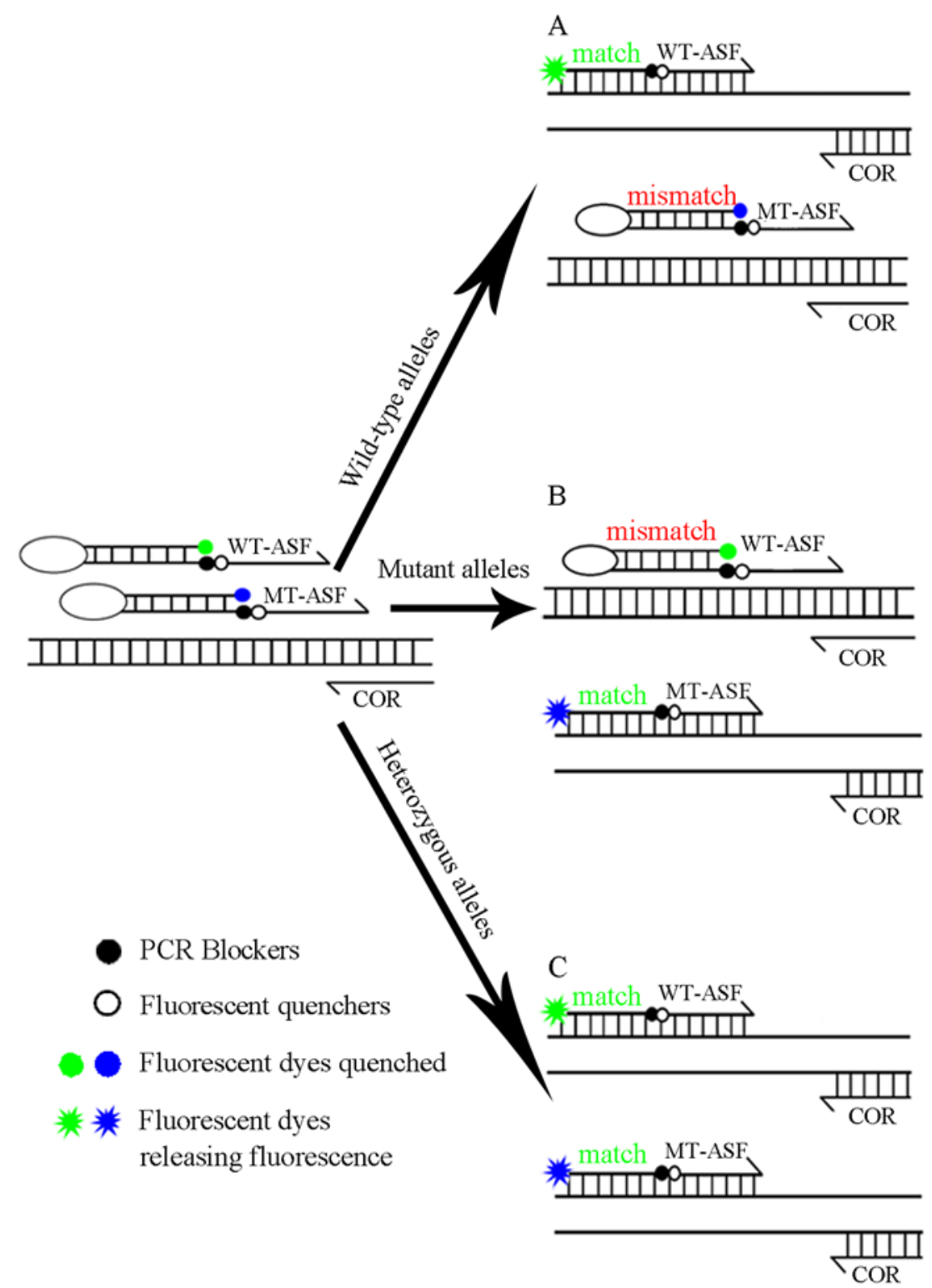

$\mathrm{D}$

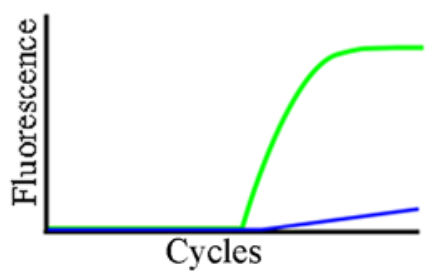

$\mathrm{E}$

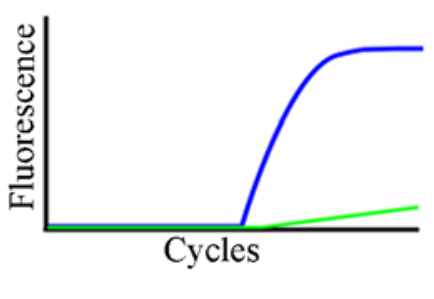

F

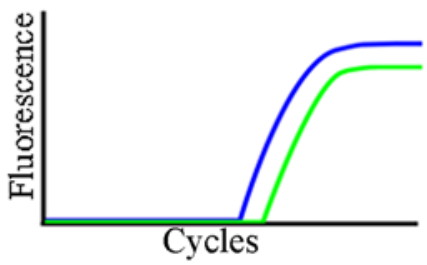

Figure 1. CRAS-PCR schematic diagram for TPMT*3B. TPMT*3B polymorphism detected by WT-ASF and MT-ASF Scorpion primers and a COR primer. The central image depicts the process of (A) WT, (B) MT and (C) mixed plasmid pairings with primers. The images on the right show the predicted amplification curves for (D) WT homozygous, (E) MT homozygous and (F) WT/MT heterozygous. The green (VIC fluorescent channel) and blue (FAM fluorescent channel) lines indicate the amplification signals of WT and MT alleles, respectively. WT, wild-type; MT, mutant; ASF, allele-specific forward; COR, common reverselCRAS-PCR, competitive real-time fluorescent allele-specific-PCR; TPMT, thiopurine S-methyltransferase; MIX, mixed.

prepare the WT-QC plasmids for TPMT*3B and TPMT*3C, respectively. The plasmids were further used to prepare a corresponding MT-QC by site-directed mutagenesis; the 141st base guanine in exon 7 was mutated into adenine (TPMT*3B, G460A) and the 147th base adenine in exon 10 was mutated into guanine (TPMT*3C, A719G). All plasmids were sequenced to confirm the TPMT*3B and TPMT*3C genotypes (Figs. S1-S4).

Evaluation of the CRAS-PCR system using TPMT*3B and $* 3 C$ QC-plasmids. Based on a previous study of duplex-crossed allele-specific-PCR (30), where TPMT*3B and $* 3 \mathrm{C}$ were identified successfully, Platinum ${ }^{\circledR}$ Quantitative PCR Supermix-UDG was tested as the reaction buffer. No amplification curves were observed in WT, MT or a MIX plasmid reaction system, for either TPMT*3B or TPMT*3C
(Fig. S5; Appendix S1). Premix Ex Taq ${ }^{\mathrm{TM}}$ Hot Start was also tested as reaction buffer; once again, gene polymorphisms could not be distinguished from a single reaction tube (Fig. S6). Therefore, Premix Ex Taq ${ }^{\mathrm{TM}}$ (Probe qPCR), which was previously used for successfully genotyping TPMT*2 (19), was used for TPMT*3B and TPMT*3C genotyping in the present study. TPMT*3B and *3C QC-plasmids were used to test the specificity of Scorpion primers targeting WT/MT TPMT*3B and *3C alleles. The amplification system contained WT and MT primers simultaneously that only had mononucleotide differences between the defined and opposite genotype primers. Although non-specific amplification was observed in the TPMT*3B and TPMT*3C QC plasmid amplification reactions, the experimental judgment was not affected (Fig. 3). The positive control results showed that Scorpion primers were able to identify TPMT $* 3 \mathrm{~B}$ and $* 3 \mathrm{C}$ 


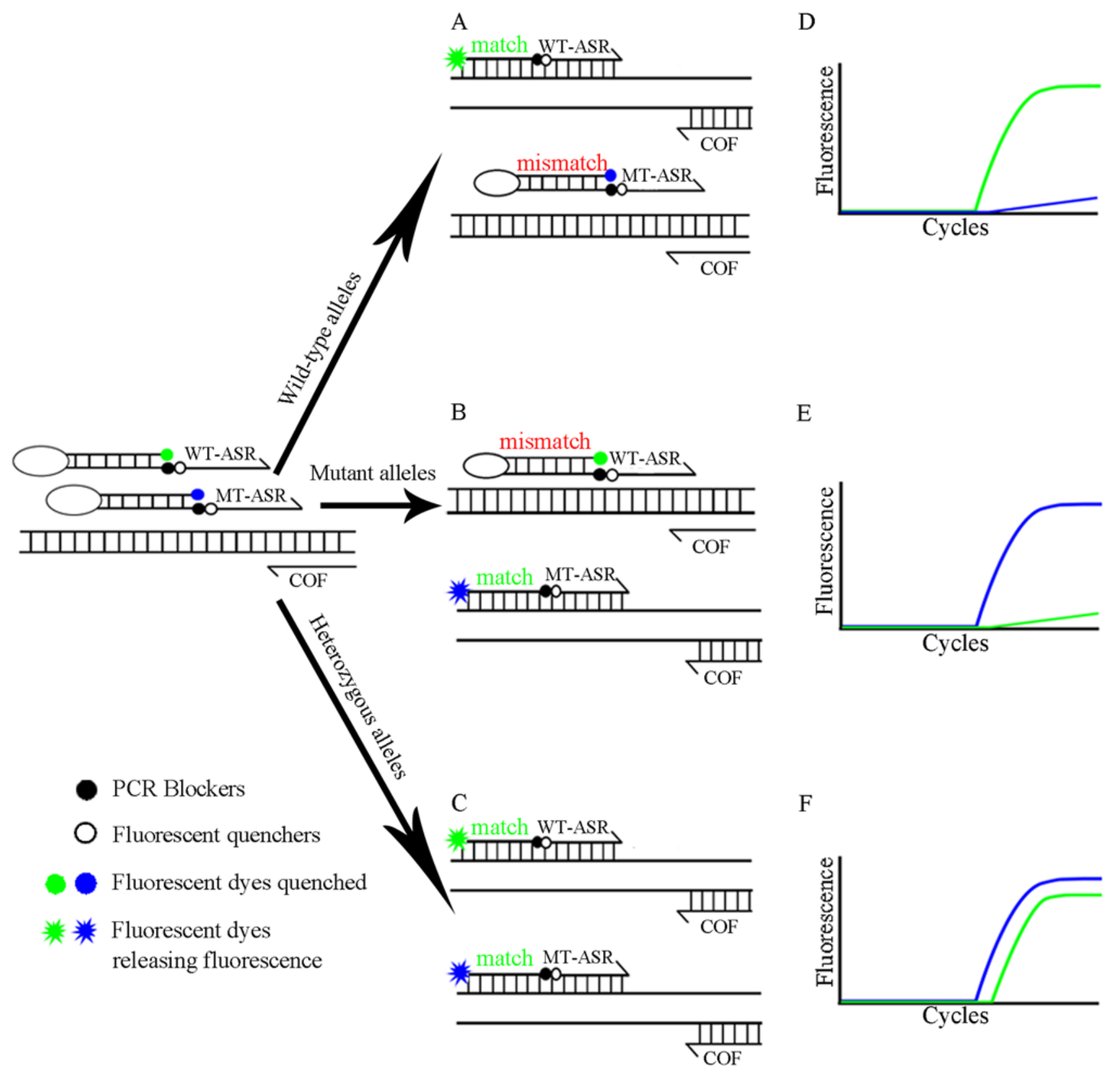

Figure 2. CRAS-PCR schematic diagram for TPMT*3C. TPMT*3C polymorphisms were detected by WT-ASR and MT-ASR Scorpion primers and a COF primer. The central image depicts the process of (A) WT, (B) MT and (C) mixed plasmid pairings with primers. The images on the right show the predicted amplification curves for (D) WT homozygous, (E) MT homozygous and (F) WT/MT heterozygous. The green (VIC fluorescent channel) and blue (FAM fluorescent channel) lines indicate the amplification signals of WT and MT alleles, respectively. WT, wild-type; MT, mutant; ASR, allele-specific reverse; COF, common forward; CRAS-PCR, competitive real-time fluorescent allele-specific-PCR; TPMT, thiopurine S-methyltransferase.

polymorphisms specifically. When the WT plasmid was used in the reaction system, the $\triangle \mathrm{Cq}$ value between FAM and VIC was found to be $\geq 3$, and the relative fluorescence units (RFU) value of VIC was three times that of FAM for TMPT*3B (Fig. 3A) and TPMT*3C (Fig. 3D). In the case of the MT plasmid in the reaction system, the $\Delta \mathrm{Cq}$ value between VIC and FAM was $\geq 3$ and the RFU value of FAM was three times that of VIC for TPMT*3B (Fig. 3B) and TPMP*3C (Fig. 3E). MIX plasmid demonstrated equal $\mathrm{Cq}$ values of FAM and VIC and equal RFU values in both TPMT*3B (Fig. 3C) and TPMT*3C (Fig. 3F). In summary, the Scorpion primers in our CRAS-PCR system could identify WT, MT and heterozygous genotypes of TPMT*3B and $* 3 \mathrm{C}$.
Analysis of blood samples from volunteers using CRAS-PCR. TPMT*3B and TPMT*3C frequencies were analyzed using the CRAS-PCR system; the reaction system was used to analyze the TPMT*3B and TPMT*3C genotypes of 226 blood samples from individuals of Han Chinese ethnicity living in Chongqing. To analyze TPMT*3B or TPMT*3C, each sample was analyzed in duplicate, and WT-, MT- and MIX-QC plasmids were tested in parallel to monitor the amplification quality. In this study, the rule that if both MT-TPMT*3B and MT-TPMT*3C were detected in the sample, the sample was identified as a variant of TPMT*3A was adopted (31).

The results revealed that of the 226 samples, all were WT homozygous for TPMT*3A and *3B (Fig. 4A). A total of 217 
A

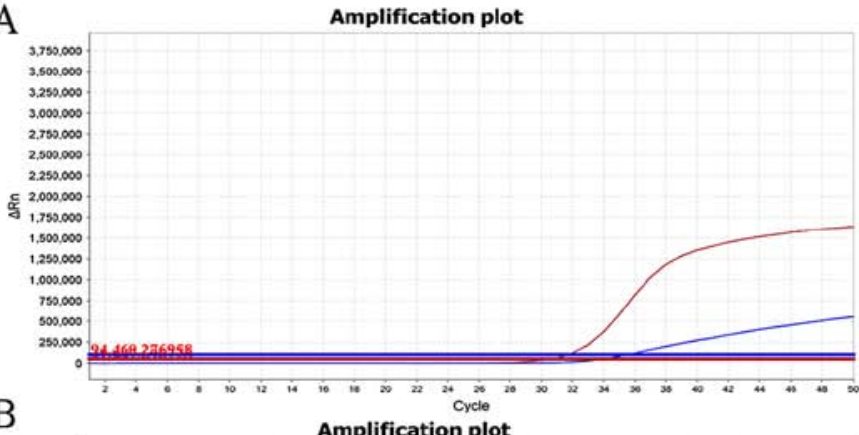

B

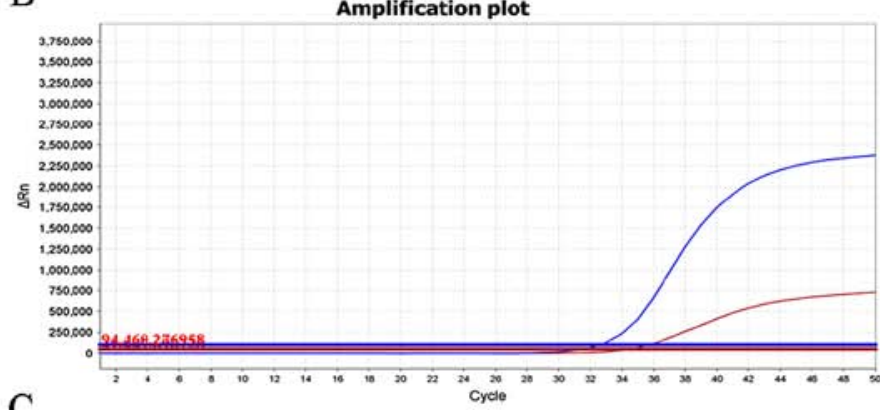

C

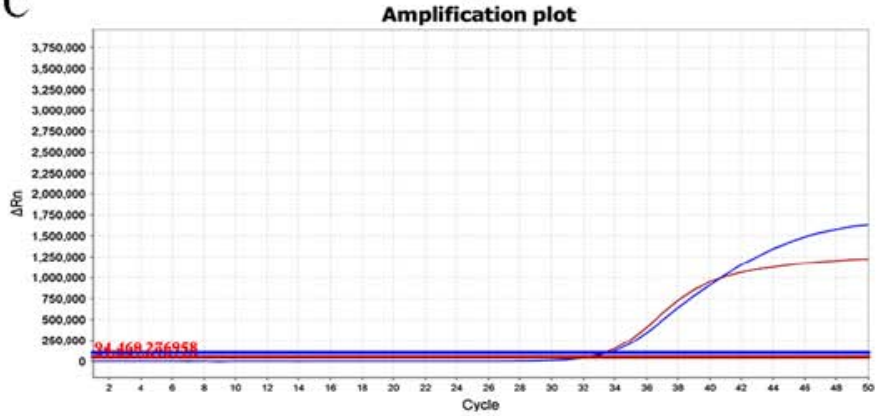

D

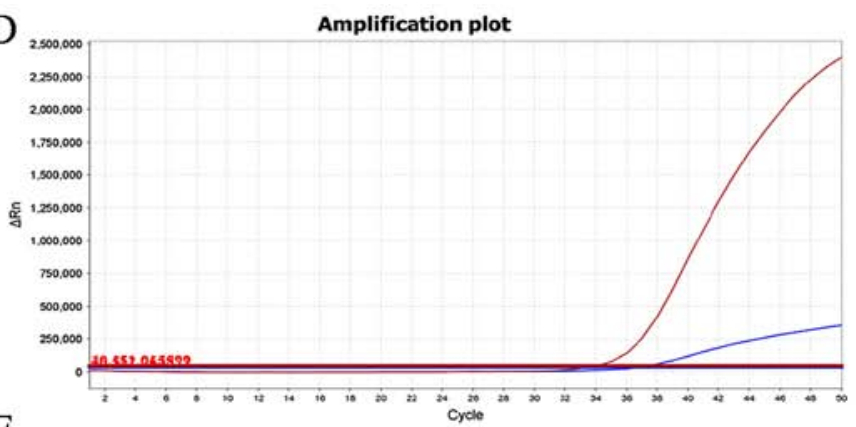

E

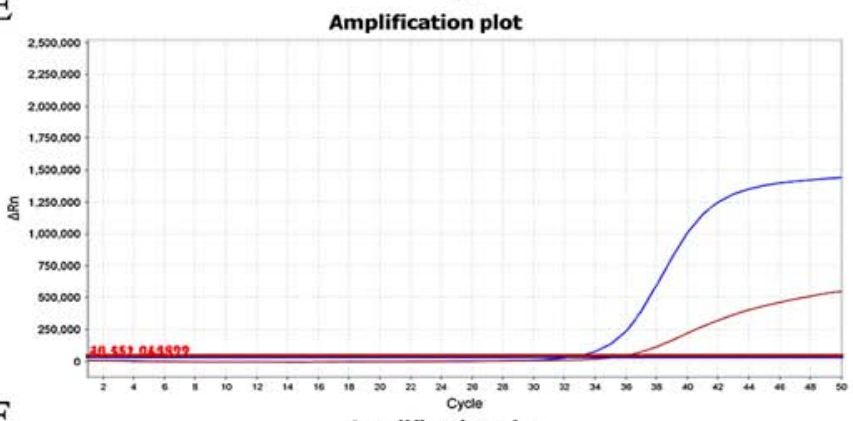

$\mathrm{F}$

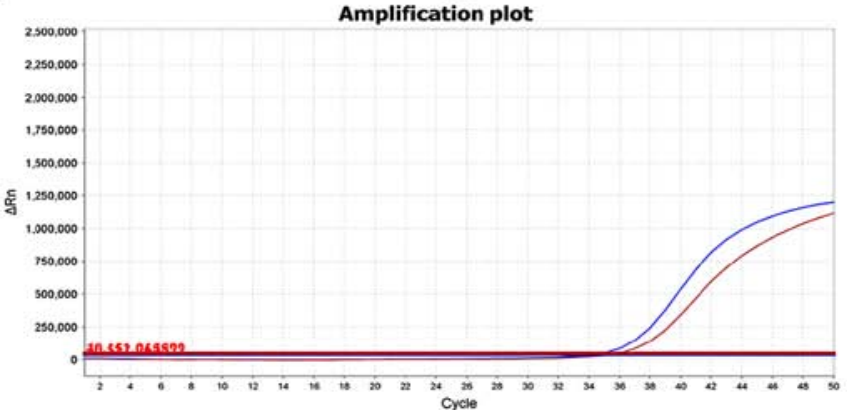

Figure 3. Amplification curves of TPMT*3B and TPMT*3C QC plasmids. (A and D) WT homozygous, (B and E) MT homozygous and (C and F) WT/MT heterozygous for TPMT*3B and TPMT*3C, respectively. The VIC fluorescence (red lines) indicates WT genotype signals and FAM fluorescence (blue lines) indicates MT genotype signals. QC, quality control; WT, wild type; MT, mutant; TPMT, Thiopurine methyltransferase.
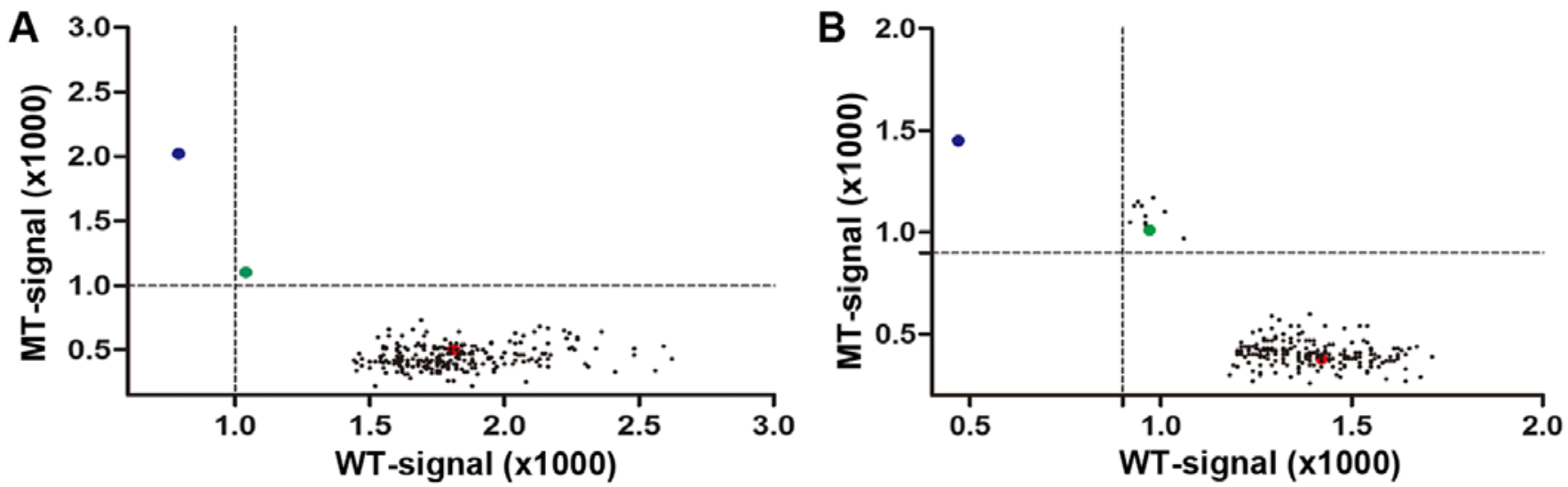

Figure 4. Mutation frequency of TPMT*3B and TPMT*3C. WT-, MT- and MIX-QC plasmids are indicated by red, blue and green dots, respectively. (A) TPMT*3B samples were all WT homozygous, (B) the mutation frequency of WT TPMT*3C homozygous accounted for 96.02\% (217/226), whereas MT heterozygous accounted for 3.98\% (9/226) and MT homozygous was not observed. WT, wild-type; MT, mutant; QC, quality control; TPMT, Thiopurine methyltransferase.

samples were WT homozygous (96.02\%), nine (3.98\%) were MT heterozygous and no samples were MT homozygous (Fig. 4B) for TPMT*3C. A mutation frequency of $3.98 \%$ is higher than that discovered in other published work for the
Han Chinese population (32). Three cases of TPMT*3C MT heterozygotes were randomly selected for DNA sequencing, and the sequencing results were consistent with our CRAS-PCR results (Fig. 5). 
A 210
CIIIIIGAAA.AIIAIA]
WT-QC plasmid

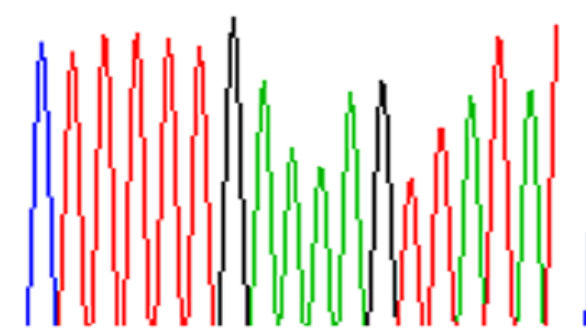

B

290 290
$\mathrm{C}$

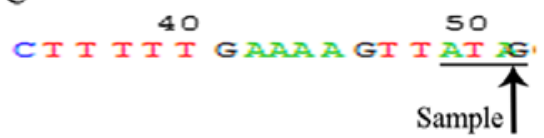

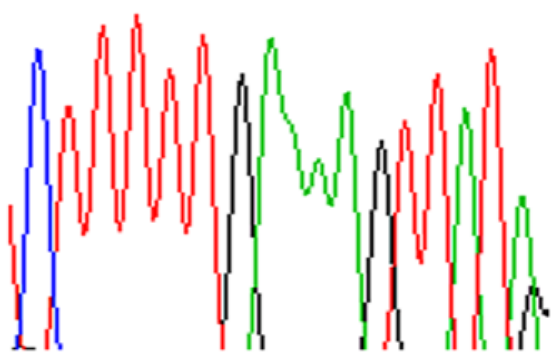

Figure 5. Sequencing analysis of a MT-TPMT*3C heterozygous sample. (A and B) Sequencing chromatogram images of WT- and MT-QC plasmid. (C) Sequencing chromatogram image of a MT heterozygous sample. The locations of MT bases are indicated with an arrow, and the underlined bases show the genetic codon containing MT bases for MT-TPMT*3C. WT, wild-type; MT, mutant; QC, quality control; TPMT, thiopurine S-methyltransferase.

\section{Discussion}

TPMT plays a crucial role in the metabolism of thiopurine drugs. Before treatment, TPMT genotyping is an important step for avoiding adverse reactions. In this study, a new method for detecting polymorphisms in TPMT*3B and TPMT*3C was explored, CRAS-PCR. The primers for TPMT*3B and TPMT*3C were designed using the WASP principle (25), with the 3 '-end terminal codon indicating allele-distinguishable variants, and the codon in the penultimate position indicating a mismatch introduced to increase primer specificity. Although non-specific amplification was still present in this study, the genotype variants were correctly identified through prior optimization of the reaction conditions.

When establishing CRAS-PCR to identify TPMT*3B and $* 3 \mathrm{C}$, the annealing temperature, number of amplification cycles, and template amount were all systematically optimized in this study (data not shown). Different annealing temperatures may alter primer-binding kinetics in a reaction mixture, and influence amplification curves and RFU values (33). In general, the annealing temperature was $55-65^{\circ} \mathrm{C}$, but when the temperature was $<57^{\circ} \mathrm{C}$ or $>63.3^{\circ} \mathrm{C}$, the specific amplification and non-specific amplification curves yielded the same $\mathrm{Cq}$ and RFU value, thus the results could not be assessed accurately. However, when the temperature was within $57-63.3^{\circ} \mathrm{C}$, the differences between $\mathrm{Cq}$ and RFU values for specific and non-specific amplification were relatively clear; therefore, an annealing temperature of $59^{\circ} \mathrm{C}$ was chosen for this reaction system, as the $\triangle \mathrm{Cq}$ and RFU ratios were clearly different between specific and non-specific amplification and allowed for interpretation of the results. Through a gradient screening of amplification of $10^{4}-10^{9}$ plasmid copies, it was found that with $10^{6}$ copies of plasmids, the $\mathrm{Cq}$ value and RFU values were consistent with that of $100 \mathrm{ng}$ genomic DNA. Therefore, a positive control concentration of $10^{6}$ copies plasmids was selected. Under these conditions, the Cq value was 32-36, and the amplification was stopped after the amplification curve became stable. To identify genetic polymorphisms accurately, the total number of cycles was set to 50 in our PCR amplification procedure.
At present, $>30$ SNPs of TPMT genes have been identified globally, and the main MT genotypes of TPMT are TPMT $* 1 / * 2$, TPMT $* 1 / * 3 \mathrm{C}$, TPMT $* 1 / * 3 \mathrm{~B}$ and $\mathrm{TPMT}^{*} 1 / * 3 \mathrm{~A}$, which account for $~ 90 \%$ of the known variant genotypes of this gene $(13,17,31,34,35)$. In Caucasian, African and Asian populations, the overall prevalence of TPMT loss-of-function variants is $0.2-0.4 \%$ for TPMT $* 2$, up to $2.6 \%$ for TPMT*3B, $1.2-6.2 \%$ for TPMT*3C and up to $5.7 \%$ for TPMT*3A $(31,36,37)$, with the TPMT $* 3 \mathrm{C}$ variant being predominant. In the Han Chinese population, TPMT $* 3 \mathrm{C}$ alleles are $1.4-3.2 \%$, but TPMT*3B and TPMT*3A alleles have not been detected $(32,38-40)$. In the present study, CRAS-PCR was used to detect TPMT*3 mutations in 226 Han Chinese individuals living in Chongqing. Mutation detection results showed that the frequencies were $3.98 \%$ for TPMT*3C, which was higher than other published results for Han Chinese populations (32). The TPMT*3A and TPMT*3B mutations were not found, which was consistent with published data on the Han Chinese population (32).

The main limitation of this study was that due to the rarity of these alleles in the test population, as well as the limited number of samples, it was not possible to validate the TPMT*3A or TPMT*3B MTs or the homozygous TPMT*3C MT. However, Sanger sequencing for TPMT*3C confirmed that the sample limitation did not affect the interpretation or validity of the results. As such, it was extrapolated that the TPMT*3C mutation was the dominant genotype in the Han Chinese population living in the Chongqing territory. This further verified that CRAS-PCR could identify TPMT*3B, *3C and *3A mutations successfully.

A simple and specific CRAS-PCR assay was established and optimized herein for the commonly found MT alleles of TPMT*3A (G460A and A719G), TPMT*3B (G460A) and TPMT*3C (A719G). The presented results confirmed that CRAS-PCR was an accurate, time-saving and simple method for the detection of mutations in TPMT*3, and may be applied in clinical trials of TPMT*3 genotyping. Further prospective studies may establish the roles of TPMT*3 in genotype-informed dosage adjustment of thiopurines, the use of alternative agents in selected patient subgroups, and the prevention of morbidity and mortality due to thiopurine intolerance. 


\section{Acknowledgements}

The authors would like to thank Sangon Biotech (Shanghai) Co., Ltd., for experimental assistance in DNA sequencing.

\section{Funding}

Professor Qing Huang received support from the Chongqing Social and People's Livelihood Science and Technology Innovation Special Project (grant no. cstc2015shmszx120102) and Major Military Logistics Scientific Research Projects (grant no. AWS17J010) for the present study.

\section{Availability of data and materials}

The datasets used and/or analyzed in the present study are available from the corresponding authors upon reasonable request.

\section{Authors' contributions}

QH planned and managed the project. NZ, BBW and LC recruited volunteers. QH, PY, XMQ, NS, LW, NZ, BBW, XDR and SR were involved in designing the study, performing the experiments and collecting the data. PY, XMQ, LC, FJS and $\mathrm{QH}$ conducted statistical analyses, interpreted the results, searched the literature and wrote parts of the manuscript. All authors read and approved the final manuscript.

\section{Ethics approval and consent to participate}

This study was carried out in accordance with the Declaration of Helsinki and was approved by the ethics committee of Southwest Hospital (Chongqing, China). All individuals signed informed consent documents.

\section{Patient consent for publication}

Not applicable.

\section{Competing interests}

The authors declare that they have no competing interests.

\section{References}

1. Appell M, Hindorf U, Almer S and Peterson C: Thiopurines in inflammatory bowel disease-the role of pharmacogenetics and therapeutic drug monitoring. Curr Pharmacogenomics 4: 285-300, 2006

2. Ford LT and Berg JD: Thiopurine S-methyltransferase (TPMT) assessment prior to starting thiopurine drug treatment; A pharmacogenomic test whose time has come. J Clin Pathol 63 288-295, 2010

3. Florin THJ, Wright JD, Jambhrunkar SD, Henman MG and Popat A: A well-tolerated and rapidly acting thiopurine for IBD? Drug Discov Today 24: 37-41, 2019.

4. Saibeni S, Kohn A, Meucci G and Papi C; Italian Group for Inflammatory Bowel Disease: How thiopurines are used for the treatment of inflammatory bowel diseases: An Italian survey. Dig Liver Dis 47: 170-173, 2015.

5. Estlin EJ: Continuing therapy for childhood acute lymphoblastic leukaemia: Clinical and cellular pharmacology of methotrexate, 6-mercaptopurine and 6-thioguanine. Cancer Treat Rev 27: 351-363, 2001.
6. Sobiak J, Skalska-Sadowska J, Chrzanowska M, Resztak M, Koltan S, Wysocki M and Wachowiak J: Thiopurine methyltransferase activity in children with acute myeloid leukemia. Oncol Lett 16: 4699-4706, 2018

7. Hollander AA, van Saase JL, Kootte AM, van Dorp WT, van Bockel HJ, van Es LA and van der Woude FJ: Beneficial effects of conversion from cyclosporin to azathioprine after kidney transplantation. Lancet 345: 610-614, 1995.

8. Hogarth LA, Redfern CP, Teodoridis JM, Hall AG, Anderson H, Case MC and Coulthard SA: The effect of thiopurine drugs on DNA methylation in relation to TPMT expression. Biochem Pharmacol 76: 1024-1035, 2008.

9. Larussa T, Suraci E, Lentini M, Nazionale I, Gallo L, Abenavoli L, Imeneo M, Costanzo FS, Cuda G and Luzza F: High prevalence of polymorphism and low activity of thiopurine methyltransferase in patients with inflammatory bowel disease. Eur J Intern Med 23: 273-277, 2012.

10. Matalon ST, Ornoy A and Lishner M: Review of the potential effects of three commonly used antineoplastic and immunosuppressive drugs (cyclophosphamide, azathioprine, doxorubicin on the embryo and placenta). Reprod Toxicol 18: 219-230, 2004.

11. Dervieux T, Blanco JG, Krynetski EY, Vanin EF, Roussel MF and Relling MV: Differing contribution of thiopurine methyltransferase to mercaptopurine versus thioguanine effects in human leukemic cells. Cancer Res 61: 5810-5816, 2001.

12. Coulthard SA, Hogarth LA, Little M, Matheson EC, Redfern CPF, Minto L and Hall AG: The effect of thiopurine methyltransferase expression on sensitivity to thiopurine drugs. Mol Pharmacol 62: 102-109, 2002.

13. Huang RS and Ratain MJ: Pharmacogenetics and pharmacogenomics of anticancer agents. CA Cancer J Clin 59: 42-55, 2009.

14. Lorenz M, Weise A, Prause S, Klemm M, Eidens M, Luchi M, Forst T, Pfützner A and Weber MM: Development and validation of a rapid and reliable method for TPMT genotyping using real-time PCR. Clin Lab 58: 959-971, 2012.

15. Maitland ML, Vasisht K and Ratain MJ: TPMT, UGT1A1 and DPYD: Genotyping to ensure safer cancer therapy? Trends Pharmacol Sci 27: 432-437, 2006.

16. Nguyen CM, Mendes MAS and Ma JD: Thiopurine methyltransferase (TPMT) genotyping to predict myelosuppression risk. PLoS Current 3: RRN1236, 2011.

17. Hindorf $U$ and Appell ML: Genotyping should be considered the primary choice for pre-treatment evaluation of thiopurine methyltransferase function. J Crohns Colitis 6: 655-659, 2012.

18. Relling MV, Gardner EE, Sandborn WJ, Schmiegelow K, Pui CH, Yee SW, Stein CM, Carrillo M, Evans WE and Klein TE; Clinical Pharmacogenetics Implementation Consortium: Clinical Pharmacogenetics Implementation Consortium guidelines for thiopurine methyltransferase genotype and thiopurine dosing. Clin Pharmacol Ther 89: 387-391, 2011.

19. Chen D, Yang Z, Xia H, Huang JF, Zhang Y, Jiang TN, Wang GY, Chuai ZR, Fu WL and Huang Q: Enhanced specificity of TPMT*2 genotyping using unidirectional wild-type and mutant allele-specific scorpion primers in a single tube. PLoS One 9: e91824, 2014.

20. Sanger F, Nicklen S and Coulson AR: DNA sequencing with chain-terminating inhibitors. Proc Natl Acad Sci USA 74: 5463-5467, 1977.

21. Román M, Cabaleiro T, Ochoa D, Novalbos J, Chaparro M, Gisbert JP and Abad-Santos F: Validation of a genotyping method for analysis of TPMT polymorphisms. Clin Ther 34: 878-884, 2012.

22. Shendure J, Porreca G, Reppas N, Lin X, McCutcheon J, Rosenbaum A, Wang M, Zhang K, Mitra R and Church G: Accurate multiplex polony sequencing of an evolved bacterial genome. Science 309: 1728-1732, 2005.

23. Shendure J, Mitra RD, Varma C and Church GM: Advanced sequencing technologies: Methods and goals. Nat Rev Genet 5: 335-344, 2004.

24. Shendure J and Ji H: Next-generation DNA sequencing. Nat Biotechnol 26: 1135-1145, 2008.

25. Wangkumhang P, Chaichoompu K, Ngamphiw C, Ruangrit U, Chanprasert J, Assawamakin A and Tongsima S: WASP: A Web-based Allele-Specific PCR assay designing tool for detecting SNPs and mutations. BMC Genomics 8: 275, 2007.

26. Collie-Duguid ES, Pritchard SC, Powrie RH, Sludden J, Collier DA, Li T and McLeod HL: The frequency and distribution of thiopurine methyltransferase alleles in Caucasian and Asian populations. Pharmacogenetics 9: 37-42, 1999. 
27. Tyagi S, Bratu DP and Kramer FR: Multicolor molecular beacons for allele discrimination. Nat Biotechnol 16: 49-53, 1998.

28. Ng CT, Gilchrist CA, Lane A, Roy S, Haque R and Houpt ER Multiplex real-time PCR assay using Scorpion probes and DNA capture for genotype-specific detection of Giardia lamblia on fecal samples. J Clin Microbiol 43: 1256-1260, 2005.

29. Wang K, Niu Y, Wang Q, Liu H, Jin Y and Zhang S: Cloning and evaluation of reference genes for quantitative real-time PCR analysis in Amorphophallus. PeerJ 5: e3260, 2017.

30. Qu XM, Zhao N, Mo QY, Yao P, Su N, Wei K, Wang L, Huang JF, Ren XD, Ren S, et al: Development of duplex-crossed allele-specific PCR targeting of TPMT*3B and *3C using crossed allele-specific blockers to eliminate non-specific amplification. Anal Biochem 575: 54-62, 2019.

31. Burchard PR, AbouTayoun AN, Lefferts JA, Lewis LD Tsongalis GJ and Cervinski MA: Development of a rapid clinical TPMT genotyping assay. Clin Biochem 47: 126-129, 2014.

32. Zhang JP, Zhou SF, Chen X and Huang M: Determination of intra-ethnic differences in the polymorphisms of thiopurine S-methyltransferase in Chinese. Clin Chim Acta 365: 337-341, 2006.

33. Lueders T and Friedrich MW: Evaluation of PCR amplification bias by terminal restriction fragment length polymorphism analysis of small-subunit rRNA and morA genes by using defined template mixtures of methanogenic pure cultures and soil DNA extracts. Appl Environ Microbiol 69: 320-326, 2003.

34. Cabaleiro T, Roman M, Gisbert JP and Abad-Santos F: Utility of assessing thiopurine $\mathrm{S}$-methyltransferase polymorphisms before azathioprine therapy. Curr Drug Metab 13: 1277-1293, 2012.
35. Robert J, Morvan VL, Smith D, Pourquier P and Bonnet J: Predicting drug response and toxicity based on gene polymorphisms. Crit Rev Oncol Hematol 54: 171-196, 2005.

36. Hamdy SI, Hiratsuka M, Narahara K, Endo N, El-Enany M, Moursi N, Ahmed MSE and Mizugaki M: Genotype and allele frequencies of TPMT, NAT2, GST, SULT1A1 and MDR-1 in the Egyptian population. Br J Clin Pharmacol 55: 560-569, 2003.

37. Teresa C, Manuel R, Javier PG and Francisco AS: Utility of assessing Thiopurine S-methyltransferase polymorphisms before azathioprine therapy. Curr Drug Metab 13: 1277-1293, 2012.

38. Kham SK, Tan PL, Tay AH, Heng CK, Yeoh AE and Quah TC: Thiopurine methyltransferase polymorphisms in a multiracial asian population and children with acute lymphoblastic leukemia. J Pediatr Hematol Oncol 24: 353-359, 2002.

39. Zhang LR, Song DK, Zhang W, Zhao J, Jia LJ and Xing DL: Efficient screening method of the thiopurine methyltransferase polymorphisms for patients considering taking thiopurine drugs in a Chinese Han population in Henan Province (central China). Clinica Chimica Acta 376: 45-51, 2007.

40. Liu L, Yang L, Zhang YC, Ai XF, Wang JX and Xiao ZJ: Polymorphisms of drug-metabolizing enzymes genes in a Han Chinese population. Zhonghua Yi Xue Za Zhi 89: 2675-2681, 2009 (In Chinese)

This work is licensed under a Creative Commons Attribution-NonCommercial-NoDerivatives 4.0 International (CC BY-NC-ND 4.0) License. 